\title{
IMPACTO DE LAS DESIGUALDADES DE GÉNERO EN EL GOCE DEL DERECHO A LA SALUD SIN DISCRIMINACIÓN
}

\author{
Pamela Eguiguren Bravo
}




\section{PAMELA EGUIGUREN BRAVO}

Matrona de la Universidad de Chile, magíster en Salud Pública y Gestión Sanitaria de la Escuela Andaluza de Salud Pública, Granada-España, y doctora en Salud Pública de la Universidad de Chile. Docente e investigadora del programa de Salud y Comunidad de la Escuela de Salud Pública Dr. Salvador Allende G. de la Facultad de Medicina de la Universidad de Chile. Integrante del Observatorio de Equidad de Género en Salud. Feminista, sus temas de estudio giran en torno a las desigualdades de género en salud y al estudio de integración de redes de atención de salud. 


\section{IMPACTO DE LAS DESIGUALDADES DE GÉNERO EN EL GOCE DEL DERECHO A LA SALUD SIN DISCRIMINACIÓN}

La movilización feminista se ha tomado el espacio y el debate universitario en estos meses, gatillando un sinnúmero de preguntas respecto a las desigualdades de género en el país, las que se viven al interior de la universidad y las que observa la sociedad chilena en su conjunto. Como nunca, las disciplinas en las distintas facultades de la Universidad de Chile están inquietas al unísono, las relaciones de género están en vitrina y el feminismo académico se hace más visible. Hay mucho aún por responder, muchos vacíos que llenar y sobre todo, mucho que comunicar. El movimiento ha dejado claras, entre muchas otras, dos cosas fundamentales: la primera es que ha desnudado el acoso sexual y su entramado de normalización en el espacio universitario y lo ha comunicado con fuerza al resto de la sociedad. Ha ensanchado así el camino para su cuestionamiento en cada espacio de interacción social, multiplicando masa crítica y agenciamiento para la rebeldía. En segundo lugar, ha vinculado este fenómeno a las desigualdades de género estructurales, aquellas que tienen su expresión en restricciones de la libertad de las mujeres en todas las esferas de la vida, en el contexto de la organización patriarcal de nuestra sociedad.

En el ámbito de la salud pública, el tema no es nuevo; las desigualdades de género en salud comienzan a estudiarse como tales desde hace al menos tres décadas ${ }^{1}$, progresivamente y con más intensidad, pero la aplicación de la perspectiva de género en la salud pública chilena y más claramente los estudios feministas, son escasos. Es un hecho reconocido tanto por la disciplina a nivel internacional como por el sistema de salud chileno y su institucionalidad que género es un determinante social estructural de la salud ${ }^{2}$, sin embargo, la incorporación de este concepto en los marcos programáticos y normativas de la institucionalidad pública de salud no ha tenido mayor expresión en la disminución de las desigualdades de género en salud y en más equidad. La evidencia advierte que a pesar de avances programáticos,

1. VERBRUGGE, L.M. Gender and health: an update on hypotheses and evidence. Journal of Health and Social Behaviour (26) 156-182.

2. MARMOT, M. ed. Subsanar las desigualdades en una generación: Alcanzar la equidad sanitaria actuando sobre los determinantes sociales de la salud. Comisión sobre Determinantes Sociales de la Salud. Resumen analítico del informe final. Ginebra: Organización Mundial de la Salud, 2008. [en línea] <http://whqlibdoc.who.int/hq/2008/WHO_IER_CSDH_08.1_spa.pdf > 
las brechas se mantienen con pocas variaciones ${ }^{3}$. En ese contexto, y a modo de contribución a la comprensión de lo necesario para estos avances, cabe hacerse la siguiente pregunta:

En un marco de evidente (re)producción de desigualdades de género en Chile, ¿es posible garantizar el derecho a la protección de la salud sin discriminación de género? Esta pregunta en referencia a los y las ciudadanos/as chilenos/s no ocurre en el vacío, por lo que habrá que indagar primero ¿cómo se expresa y cuál es el significado y contenido de este derecho en nuestra Constitución política?

\section{EL DERECHO A LA PROTECCIÓN DE LA SALUD}

La salud es un derecho humano fundamental, es un hecho que involucra una complejidad de aspectos y que va más allá de la valoración en un determinado momento sobre estar o sentirse sana/o, en ausencia de una enfermedad. La definición fundante de salud de la Organización Mundial de la Salud (OMS) ${ }^{4}$, "un estado de completo bienestar físico, mental y social, y no sólo la ausencia de enfermedad", guía el modelo biopsicosocial de atención de salud chileno desde hace varias décadas, aun cuando cabe destacar que para varios autores esa definición no recoge de manera adecuada el dinamismo de los procesos de salud-enfermedad y de las condiciones y relaciones sociales en las que se construye colectivamente la salud ${ }^{5}$. La constatación de esta complejidad hace difícil precisar lo que el derecho a la salud contiene y hablar de derecho impone la necesidad de significar lo que al Estado cabe respetar, proteger y promover ${ }^{6}$. Esa discusión enfrentó la comisión redactora de la Constitución Política que nos rige actualmente -recordemos que se trata de la Constitución de 1980, redactada en plena dictadura- y según algunos/as autores/as, la intención habría sido restringirlo al acceso a servicios. Por ello, su articulado no aludiría al "derecho a la salud" sino al "derecho a la protección de la salud" y en su bajada más específica, al acceso igualitario a acciones, pero más allá de la intencionalidad, ¿cuál sería la diferencia? En una revisión desde el derecho publicada por Figueroa ${ }^{7}$ (2013) se destaca cómo para

3. INDH. Informe Anual 2017. [en línea] <https://www.indh.cl/bb/wp-content/ uploads/2017/12/01_Informe-Anual-2017.pdf>

4. OMS, Definición adoptada en el marco de la Conferencia Internacional de la Salud NY 1946.

5. NAVARrO, V. Concepto actual de la salud pública. En Martínez, F., Castellanos, P. L., Navarro, V., Salud Pública. Ciudad de México: Mc Graw-Hill. 1998.

6. NACIONES UNIDAS: Conceptos clave sobre los DESC - ¿Cuáles son las obligaciones de los Estados respecto de los derechos económicos, sociales y culturales? [en línea] <https://www. ohchr.org/SP/Issues/ESCR/Pages/WhataretheobligationsofStatesonESCR.aspX>

7. FIGUEROA GARCÍA HUIDOBRO, R. El derecho a la salud. Centro de Estudios Constitucionales de Chile, Universidad de Talca. Año 11(2):283-332. 2013. 
algunos autores, los significados y límites de contenido de ambos conceptos pueden moverse hacia una interpretación amplia o restrictiva, dependiendo del autor y su ideología, y llegar en algunos casos a usarse de forma si no intercambiable, al menos homologable.

Resultan centrales en la diferenciación de ambos conceptos las acciones a las cuales el Estado y sus agentes estarían comprometidos en su rol garante de los derechos constitucionales. Estar sana/o, enfermarse o recuperar la salud después de una enfermedad son fenómenos que también involucran aspectos relativos a la genética y a otras situaciones vinculadas al actuar individual y por ello no todo sería posible de garantizar por el Estado. Aunque lo anterior tiene sentido, es importante señalar que sabemos que esto tampoco puede ser completamente atribuido a las personas y sus decisiones, dadas las limitaciones que el sistema impone para enfrentar en su cotidiano la satisfacción de necesidades, las que constituyen en sí mismas derechos humanos fundamentales y muy vinculados a la salud. En ello, el Estado evidentemente tiene ámbitos de responsabilidad. Nancy Krieger ${ }^{8}$ plantea y profundiza el concepto de embodiment, donde postula que la genética, la biología y sus expresiones en el transcurso de la vida estarían imbricadas y socialmente determinadas, es decir, lo social se va haciendo cuerpo y biología; también las desigualdades de género experimentadas por el cuerpo social impactan a las personas y sus cuerpos en su individualidad. Lo anterior resulta evidente cuando se analizan desigualdades sociales en la ocurrencia de enfermedades o problemas de salud específicos, donde colectivos vulnerados tienen la mayor carga de enfermedad y los peores resultados. Cuando se vive en sociedades cuyos Estados no garantizan igualdad de derechos, los resultados de salud son indicadores de desigualdad.

Volviendo a la reflexión sobre el derecho a la salud y el derecho a la protección de la salud, en la comparación entre ambos conceptos no es tan relevante su diferencia como lo que comparten. En ese sentido, proteger el "derecho a la salud" o garantizar el "derecho a la protección de la salud" parecen no diferir en la interpretación de la obligación del Estado de proteger la salud como derecho social'. Figueroa sostiene en sus conclusiones que hay suficientes argumentos para afirmar que en nuestra Constitución el derecho a la protección es un derecho exigible ${ }^{10}$ y que aun cuando no estén expresados en el texto constitucional todos sus posibles contenidos, esto

8. KRIEGER, N. Glosario de epidemiología social. Rev. Panam. Salud Pública. 11(5-6): 10204989. 2002.

9. Tal cual está establecido en el Pacto de Derechos Sociales Económicos y Culturales - suscrito por el Estado chileno.

10. FIGUEROA GARCÍA HUIDOBRO, R. El derecho a la salud. Centro de Estudios Constitucionales de Chile, Universidad de Talca. Año 11(2):283-332. 2013. 
para nada quiere decir que la interpretación del derecho a la protección de la salud quede limitada a las enumeraciones allí plasmadas:

\section{Art. 9 de la Constitución Política de Chile: El derecho a la protección de la salud ${ }^{11}$}

El Estado protege el libre e igualitario acceso a las acciones de promoción, protección y recuperación de la saludy de rebabilitación del individuo.

Le corresponderá, asimismo, la coordinación y control de las acciones relacionadas con la salud.

Es deber preferente del Estado garantizar la ejecución de las acciones de salud, sea que se presten a través de instituciones públicas o privadas, en la forma y condiciones que determine la ley, la que podrá establecer cotizaciones obligatorias.

Cada persona tendrá el derecho a elegir el sistema de salud al que desee acogerse, sea éste estatal oprivado.

De hecho, observando el texto, el concepto de promoción de la salud tiene amplia lectura. Las acciones positivas en ese ámbito registran desde actividades y programas a nivel local hasta la elaboración e implementación a nivel país de políticas y leyes, donde la intersectorialidad es principio fundamental ${ }^{12}$.

En la reflexión sobre los márgenes de interpretación del derecho a la protección de la salud en Chile existen ejemplos en la jurisprudencia del Tribunal Constitucional de aplicabilidad de las disposiciones constitucionales, donde se han reconocido conceptos y dimensiones del derecho a la salud que están contenidos en el Pacto Internacional de Derechos Sociales, Económicos y Culturales (PIDESC) y en varios de los informes y observaciones del Comité de Derechos Económicos, Sociales y Culturales (CESCR) ${ }^{13}$. El PIDESC señala que las acciones positivas del Estado deben apuntar a garantizar para sus ciudadanas y ciudadanos "el disfrute del más alto nivel posible de salud". La interpretación del derecho en el marco del pacto es

11. Constitución Política de la República de Chile, 1980. Cap. III De los Derechos y Deberes Constitucionales.

12. OPS, BREVE GUÍA y recomendaciones para promover la colaboración intersectorial. Impulsar el enfoque de la salud en todas las políticas en las Américas: ¿Cuál es la función del sector de la salud? [en línea] <https://www.paho.org/hq/dmdocuments/2015/hiap-Brief-Guide-andRecomendations-SPA.pdf $>$

13. FIGUEROA GARCíA HUIDOBRO, R. El derecho a la salud. Centro de Estudios Constitucionales de Chile, Universidad de Talca. Año 11(2):283-332. 201. 
explícita en sus observaciones, donde se consideran acciones vinculadas a factores determinantes de la salud:

"El Comité interpreta el derecho a la salud, definido en el apartado 1 del artículo 12, como un derecho inclusivo que no sólo abarca la atención de salud oportuna y apropiada sino también los principales factores determinantes de la salud, como el acceso al agua limpia potable y a condiciones sanitarias adecuadas, el suministro adecuado de alimentos sanos, una nutrición adecuada, una vivienda adecuada, condiciones sanas en el trabajo y el medio ambiente, y acceso a la educación e información sobre cuestiones relacionadas con la salud, incluida la salud sexual y reproductiva. Otro aspecto importante es la participación de la población en todo el proceso de adopción de decisiones sobre las cuestiones relacionadas con la salud en los planos comunitario, nacional e internacional"14.

Tomando las conceptualizaciones anteriores, toca observar la realidad y las posibilidades de ejercicio del derecho a la salud en el marco político, económico, legislativo y sanitario chileno. Cabe destacar que en ese ejercicio o disfrute, el respeto, la protección y promoción, como deberes del Estado, tienen su traducción completa cuando se comprende que todos los derechos implican también libertades y el ámbito en que probablemente resulte más fácil visualizarlas es el de la autonomía sobre el propio cuerpo. Estas libertades también están reconocidas en el PIDESC.

"El derecho a la salud no debe entenderse como un derecho a estar sano. El derecho a la salud entraña libertades y derechos. Entre las libertades figura el derecho a controlar su salud y su cuerpo, con inclusión de la libertad sexual y genésica, y el derecho a no padecer injerencias, como el derecho a no ser sometido a torturas ni a tratamientos y experimentos médicos no consensuales. En cambio, entre los derechos figura el relativo a un sistema de protección de la salud que brinde a las personas oportunidades iguales para disfrutar del más alto nivel posible de salud"15.

14. El derecho al disfrute del más alto nivel posible de salud: 11/08/2000. E/C.12/2000/4, CESCR OBSERVACION GENERAL 14. (General Comments) [en línea] <http://www.acnur.org/ fileadmin/Documentos/BDL/2001/1451.pdf?view=1;El>

15. Ibidem. 


\section{LAS DESIGUALDADES DE GÉNERO EN SALUD}

El análisis de las desigualdades de género en salud se sustenta en teorías feministas y en la comprensión de un sistema sexo-género que construye determinaciones sociales diferenciadas para mujeres y hombres en función de la dicotomía hombre/ mujer (sexo) y de un correspondiente par obligado masculino/femenino (género). A la luz de la discusión teórica actual, la categoría sexo, antes pensada como dicotomía inamovible, hoy se entiende también como una construcción social -pre-discursiva a género-, ${ }^{16}$ como tantos otros binarismos instalados en la naturalización de las desigualdades y en la legitimización de una "normalidad" que da la espalda a lo diverso.

Género es una categoría de análisis y nos permite identificar y analizar de manera crítica las construcciones sociales y culturales que sostienen expectativas sobre un orden y una "normalidad" conducida a través de los roles de género y su jerarquía relacional, determinando sus espacios, sus recursos, su poder y su acceso al reconocimiento social. Su expresión social e institucional nos levanta y conduce desde el nacimiento en adelante por rutas que pocas veces son comunes entre las mujeres y los hombres, y aunque a veces compartan ciertos senderos, generalmente los recursos y las condiciones siguen haciendo distinto el camino, el esfuerzo y la llegada. Si bien la categoría género presenta limitaciones cuando se posiciona desde el binarismo masculino/femenino respecto de las diversidades actuales, no es menos cierto que estas construcciones siguen siendo vigentes para limitar los derechos de las personas que no se ajustan a ellas.

Diderichsen, Evans y Whitehead ${ }^{17}$, en el libro Desafio a la falta de equidad en salud de la Organización Panamericana de la Salud (OPS), plantean la existencia de cuatro mecanismos en la construcción social de inequidades en salud, invitando a su aplicación para comprender mejor las determinaciones sociales en salud y también los puntos de entrada de las acciones políticas y programáticas para su abordaje. Estos hacen posible levantar los muros estructurales de una división compleja que genera inequidades en salud, donde la interseccionalidad agrega y potencia inequidades y discriminaciones en grupos sociales que comparten varios ejes de

16. VARTABEDIAN, J. El cuerpo como espejo de las construcciones de género. Una aproximación a la transexualidad femenina. Quaderns-e de l'ICA, (10). 2007.

17. DIDERICHSEN, F., EVANS, T., WHITEHEAD, M. Bases sociales de las disparidades en salud. En: EVANS, T., WHITEHEAD, M., DIDERICHSEN, F., BHUIYA, A, WIRTH, M. (Eds.) Desafío a la falta de equidad en salud: de la ética a la acción. OPS, 2010. pp.13-25. 
desigualdad ${ }^{18}$. Hablar de inequidades alude al hecho de tratarse de desigualdades cuya configuración depende de la organización social, con resultados que son injustos y evitables ${ }^{19}$. Los mecanismos planteados por los autores son la estratificación social, la exposición diferencial, la vulnerabilidad diferencial y las consecuencias diferenciales sobre la salud, que impactan la vida de mujeres y hombres.

Como resulta evidente, dependemos de las condiciones sociales, históricas y políticas en las que nacemos y nos desarrollamos, lo que, de hecho, determina primariamente qué tan sujetos de derecho somos en nuestros contextos. En esa configuración importa el concepto y el nivel de desarrollo del país en el que estamos, sus leyes y sus políticas de protección social, su sistema económico y político, el acceso a los medios de producción, la existencia de mecanismos de redistribución del ingreso y recursos sociales, el poder sobre los medios de comunicación masiva y de manera muy fundamental, el respeto a los derechos humanos y su institucionalidad en el país. Estas dimensiones y otras que podrían señalarse, definen un punto de partida.

La descripción desagregada según sexo de indicadores sociales y económicos nos da el primer pie para su interpretación bajo el análisis de género, iluminando el primer mecanismo, de estratificación social. Género determina distintas oportunidades y significados en los procesos y trayectorias de vida de mujeres y hombres. Sus oportunidades, logros educativos y laborales no pueden desvincularse delos mandatos y ordenamientos de género, de su significado y valorización del trabajo (productivo/ reproductivo). En esa línea de pensamiento, la maternidad constituye un evento determinante en los proyectos de vida de las mujeres, un reflejo de ello es cómo la maternidad adolescente impacta las posibilidades de desarrollo y posición social de las mujeres. La división sexual del trabajo configura una desigual distribución de las labores domésticas y de cuidado, cuyo mandato social y limitaciones se traducen, por ejemplo, en una baja participación laboral e independencia económica de las mujeres en el caso de Chile, de las más bajas en América Latina ${ }^{20}$. Quienes tienen acceso a un trabajo remunerado se enfrentan a la segregación horizontal [de género], con distinta apertura y características en las opciones de trabajos disponibles, y a la segregación

18. DISCUSSION PAPER, Policy and Education Branch. An Intersectional Approach to Discrimination: Addressing Multiple Grounds in Human Rights Claims. Ontario Human Rights Commission, 2001 [en línea] <http://www.ohrc.on.ca/sites/default/files/attachments/An_ intersectional_approach_to_discrimination\%3A_Addressing_multiple_grounds_in_human_ rights_claims.pdf >

19. WHITEHEAD, M. The concepts and principles of equity and health. OPS, 1991

20. CEPAL, Indicadores Observatorio de Igualdad de Género ALC [en línea] <https://oig.cepal. org/es/autonomias/autonomia-economica> 
vertical, con acceso diferenciado por género a cargos e influencias en los espacios de poder, donde las mujeres son generalizadamente subordinadas. Reflejo de ello son el mayor estatus, reconocimiento social y retribuciones económicas para los trabajos, responsabilidades y desempeños masculinos. En torno a esta desigualdad estructural se ordenan otra serie de determinantes intermedios, que condicionan en múltiples planos las diferencias de acceso a poder, recursos y reconocimiento.

Con respecto al segundo mecanismo, de exposición diferencial, cabe reflexionar sobre las condiciones de vida y trabajo bajo el prisma de los roles de género, su distinto significado relacional, social y sus atribuciones. Frente a una misma enfermedad o problema de salud, mujeres y hombres están expuestos/as de forma diferenciada y también registran exposiciones diferentes, relacionadas a las construcciones de género. Pensemos en la salud mental y en un diagnóstico como la depresión, donde la medición de prevalencia (últimos 12 meses) en la última Encuesta Nacional de Salud (ENS) arrojó un 10,1\% en mujeres frente a un 2,1\% en los hombres ${ }^{21}$. Aplicando análisis de género surgen explicaciones sobre cómo distintos factores vinculados a la depresión se ordenan tras patrones de género. La depresión post-parto, la depresión de quienes cuidan y la depresión de víctimas de violación y abuso sexual están claramente asociadas a determinaciones femeninas ${ }^{22} 23$, mientras que en el caso de los hombres la relación es con exigencias impuestas por las construcciones de masculinidad, como el éxito en la provisión económica. La exposición a este problema se inscribe sin duda en las rutas vitales dibujadas desde y con género y su enfrentamiento hace visible el tercer mecanismo de producción de inequidades: la vulnerabilidad diferencial. Frente a una determinada exposición, mujeres y hombres son vulnerables en distinta forma y medida; siguiendo el caso de la depresión, culturalmente para las mujeres existe una mayor aceptación, no sin ningún costo de estigmatización, pero claramente menor que en el caso de los hombres. Aquellos, bajo construcciones de masculinidad, frente al malestar emocional buscan menos ayuda y más tarde, lo que los hace más vulnerables.

Finalmente, el cuarto mecanismo de consecuencias diferenciales trata de las implicancias o efectos de la enfermedad o problema de salud y cómo estos difieren

21. ENCUESTA NACIONAL DE SALUD Chile, 2016-2017, Segunda entrega de resultados [en línea] http://www.minsal.cl/wp-content/uploads/2018/01/2-Resultados-ENS MINSAL_31_01_2018.pdf

22. SCHULZ, R, y SHERWOOD, P, Physical and Mental Health Effects of Family Caregiving, Am J Nurs.; 108(9 Suppl): 23-27. 2008 doi:10.1097/01.NAJ.0000336406.45248.4c.

23. WISE, L., ZIERLER, S., KRIEGER, N., HARLOW, B. Adult onset of major depressive disorder in relation to early life violent victimisation: a case-control study. The Lancet; Vol 358; 881-887. 2001. 
en razón de género. El significado cultural de la depresión proyecta consecuencias sociales para unas y otros, por ejemplo, en la esfera laboral, en la estigmatización y su impacto sobre relaciones inter-personales, etc., las cuales se configurarán de manera desigual para los géneros. Aquí también adquiere relevancia, como en el primer mecanismo, la estructura y el contexto político, económico y social, pues las consecuencias podrían profundizar desigualdades, generando nuevos impactos sobre la estratificación social.

Pensar estos mecanismos ayuda también a identificar determinaciones sociales de manera más próxima a las personas, aquellas que están contenidas en sus experiencias y decisiones concretas de vida. Comprender cómo el género -colectivamente determinado- toma parte desde cada quien en sus decisiones y posibilidades, en su trayectoria vital. Cómo el género determinará el desarrollo de proyectos vitales que podrán ser más o menos planificados, autónomos, más o menos injustos, sujetos a violencias y, en consecuencia, más o menos saludables. Muchas veces habremos escuchado o sentido nosotros/as mismos/as el vértigo de aquellas decisiones fundamentales que mediaron para convertirnos en quienes somos. Revisando esas historias podemos identificar sin duda el peso que las construcciones de género han podido tener en nuestras propias vidas y sus consecuencias para nuestra salud, y visualizar las acciones efectivas de protección del Estado o su ausencia.

Cada uno de los mecanismos revisados representa niveles que se agregan en la producción de desigualdades de género, sumando y potenciando sus efectos sobre la salud. Reflexionar sobre ello aporta a la identificación de acciones a distintos niveles para desarticular la reproducción de inequidades y en suma, garantizar derechos.

\section{EL DERECHO A GOZAR DE LA SALUD SIN DISCRIMINACIÓN}

Ya hemos reflexionado sobre cómo las desigualdades de género en salud se reproducen por la vía de la estructura, organización social y la cultura, determinando diferencias injustas y evitables en las posibilidades concretas de gozar del derecho a la salud. Las inequidades de género en salud se expresan, según el siguiente ordenamiento (Gómez 2002), en distintas dimensiones ${ }^{24}$ : a. resultados de salud y sus determinantes, b. la asignación de recursos y el acceso efectivo a la atención de acuerdo con la necesidad, c. financiamiento de la atención según capacidad de pago y d. la distribución del poder y la carga de responsabilidades en el cuidado de la salud.

24. GÓMEZ, E. Género, equidad y salud: Retos para la acción, Revista Panamericana de Salud Pública, 11 (5/6), OPS, 2002. 
El primer punto pone atención sobre un conjunto de indicadores de salud y también sociales que mostrarían los efectos de las inequidades de género. Sabemos que cuando nos referimos a género también hablamos de los hombres, las desigualdades no son saludables para nadie, y en muchos casos los patrones de masculinidades hegemónicas implican daño a la salud. Sin embargo, la desigualdad estructural lleva una dirección en la que los resultados más críticos y de permanente profundización están en el género femenino, de modo que el indicador de igualdad para el goce del derecho a la salud y su protección debe partir por quebrar esta subordinación. En esa línea, se constata en nuestro país una brecha de participación laboral que no logra quebrarse, donde las mujeres tienen sustancialmente menos probabilidades de tener ingresos propios y muchas de ser responsables principales del cuidado en sus hogares. Están sobre-representadas en trabajos más precarios y en quintiles de bajos ingresos, y una persistente brecha salarial aumenta a mayor nivel de instrucción. Estos y muchos otros indicadores dan cuenta del pleno funcionamiento de las determinaciones de género antes comentadas. Junto a ello, las mujeres viven diversas expresiones de violencia de género, comenzando por sus espacios más íntimos y cotidianos, hasta los espacios de interacción con la institucionalidad. Estas desigualdades se expresan luego en resultados de salud/enfermedad, por ejemplo, en la preponderancia femenina en los trastornos depresivos y otra serie de condiciones crónicas que implican serias limitaciones para la vida plena. En la esfera de la salud sexual y reproductiva, las mujeres enfrentan grandes limitaciones para el ejercicio de sus libertades sexuales y sus decisiones reproductivas, donde se cruzan y potencian diversas violencias, como la violencia sexual y la violencia institucional.

Volviendo a las dimensiones arriba planteadas y a nuestra pregunta acerca del impacto de las desigualdades en el goce del derecho a la salud sin discriminación, cuando abordamos las otras tres dimensiones de la salud impactadas por determinaciones de género (asignación de recursos y acceso efectivo a la atención, al financiamiento según capacidad de pago y a la distribución de responsabilidades versus poder en la toma de decisiones) nos encontramos frente a un campo de acción directa del Estado, por lo que las acciones positivas de protección de la salud podrían aminorar efectos o desarticular mecanismos de producción de inequidad. Resulta claro que las acciones del Estado para respetar, proteger y promover el derecho igualitario a la protección de la salud deben ser conscientes y dirigidas a que su goce pueda realizarse sin discriminación de género, pues las políticas no son nunca neutras y distintos cursos de acción pueden significar avances o retrocesos ${ }^{25}$ en materia de equidad de género.

25. EGUIGUREN, P., CALVIN, ME., DÍAZ, X., FERRER, M., IGLESIAS, M., OLAVARRIA, J. Observatorio de equidad de género en salud - Chile: una mirada a las políticas públicas. Rev. Chilena Salud Pública [en línea]. <http://www.revistas.uchile.cl/index.php/RCSP/arti cle/ viewFile/644/542.> 
La reflexión nos lleva a pensar en las acciones del Estado en varios planos, las que representan configuraciones estructurales macro, que acumulan el registro y el peso de la historia política, como también aquellas acciones y políticas gubernamentales en un nivel meso, donde podemos observar algunas más recientes y contingentes.

En el ámbito de este análisis sobre el derecho a la salud, la estructura del sistema de salud resulta fundamental, influyendo en las tres dimensiones señaladas. Con las reformas de los años '80 (durante la dictadura cívico-militar), el sistema de salud chileno estableció una estructura dual con un subsistema público y otro privado que perdura hasta la actualidad. Las inconveniencias y desventajas de esta estructura para los principios de igualdad y solidaridad son vastamente reconocidas, sin embargo, no ha existido en los gobiernos democráticos posteriores la voluntad política para llevar adelante propuestas de cambio estructural; por el contrario, muchas de las medidas implementadas -especialmente en el gobierno de Ricardo Lagos- profundizaron las lógicas de mercado en la salud ${ }^{26}$. Las reformas en dictadura también modificaron la estructura del antiguo Servicio Nacional de Salud, generando la fragmentación de la red asistencial y la existencia de grandes desigualdades por la segmentación económica a nivel territorial con la administración municipal de la atención primaria. La transferencia de recursos financieros desde el sistema público hacia el subsistema privado, con el lucro creciente de este último y el debilitamiento del sistema público, se han transformado en datos que ya no asombran a nadie. En términos de igualdad de género, esta estructura evidentemente no se hace cargo ni corrige las desigualdades, no contribuye a la redistribución de recursos y, por el contrario, profundiza la estratificación social de género y sus inequidades en todas las dimensiones señaladas.

"Si se consideran las desventajas de las mujeres en materia de distribución de poder y recursos, es evidente que los sistemas de salud capaces de responder a sus necesidades diferenciadas deben ser solidarios, universalistas, capaces de asegurar el pleno acceso y el avance hacia la equidad de género, estrato socioeconómico y etnia, y en los cuales la toma de decisiones se efectúe en forma participativa e inclusiva"27.

26. Asociación Latinoamericana de Medicina Social (ALAMES) Chile: El derecho a la salud. En: Derecho a la Salud, Situación en países de América Latina. 2015, pp.29-39. <http://www. alames.org/index.php/documentos/libros/medicina-social/informes>

27. MATAMALA, MI., EGUiGUREN, P., DÍAZ, X. Tensiones y Silencios en la Reforma de la Salud 2011: Género y Derechos Ausentes; Observatorio de Equidad de Género en Salud-OPS; 2011. 
La dictadura también quebró a partir del golpe militar una relación virtuosa entre la producción académica en salud pública y su articulación con la política pública. Programas y políticas señeras que perduran hasta hoy surgieron de esa relación, donde la mirada social ocupaba un lugar central en la detección y análisis de los problemas y necesidades de salud de la población. En materia de salud sexual y reproductiva, por ejemplo, los estudios sobre anticoncepción y aborto realizados desde la entonces llamada Escuela de Salubridad por Tegualda Monreal y Rolando Armijo, claramente nutrieron el desarrollo de la política de planificación familiar de la década del ' 60 con acciones de un enorme impacto en el país en materia de reducción de mortalidad de mujeres y niños $/ \mathrm{as}^{28}$. Durante la dictadura, las limitaciones y retrocesos en la disponibilidad y acceso a estos servicios vulneró de manera flagrante los derechos de las mujeres, asestando su golpe final con una última ley en 1989, que eliminó el aborto terapéutico de nuestro código sanitario (de 1931) y dejó a las mujeres chilenas ante la prohibición de la realización de abortos bajo cualquier circunstancia, acceso que sólo ha sido posible reponer para tres causales en 2017.

En democracia, la incorporación de género en la mirada gubernamental se plasmó tanto en la creación del Servicio Nacional de la Mujer (hoy Ministerio de la Mujer y Equidad de Género) como en la existencia de espacios y referentes sectoriales de género, además de una serie de instrumentos para la transversalización de esta mirada y su traducción a políticas y programas. Sin embargo, estos avances fueron insuficientes para la incorporación de género en la corriente central de la política y la voluntad no alcanzó para los cambios estructurales requeridos ${ }^{29}$; la opción fue el camino de las políticas de igualdad de oportunidades, renunciando a las grandes transformaciones. En el último gobierno de Michelle Bachelet, la aprobación de la ley 21.030 tras dos años de debate logró abrir acceso mínimamente a tres causales frente a situaciones críticas en las que las mujeres tendrán la posibilidad de decidir continuar o no con sus embarazos ${ }^{30}$. Las causales acogidas representan cerca de un $3 \%$ de la estimación de demanda anual de aborto inducido que hasta antes de la ley

28. MATAMALA, MI. Aborto en Chile: cuerpos, derechos y libertades en: Voces sobre el aborto, ciudadanía de las mujeres, cuerpo y autonomía. Articulación feminista por la libertad de decidir y Escuela de Salud Pública Dr. Salvador Allende G. Facultad de Medicina de la Universidad de Chile. Santiago 2014, pp.7-20.

29. MATAMALA, MI. A medio camino en un entrevero: ¿quedó desnuda la igualdad de género? En: BUROTTO, A. y TORRES, C. (Eds). Y votamos por ella: Michelle Bachelet: miradas feministas. Fundación Instituto de la Mujer; Fundación Heinrich Böll Cono Sur. Santiago de Chile 2010, pp.141-165.

30. Ley21.030 Regulalainterrupción voluntaria delembarazoen tres causales. 23 deseptiembrede 2017 [en línea] < https:/ /www.leychile.cl/Navegar?idNorma=1108237> 
se resolvía en su totalidad en clandestinidad ${ }^{31}$. Si bien es un avance importante, su implementación efectiva y el empuje para el cambio cultural de despenalización social del aborto no está garantizado sin el debido respaldo de la institucionalidad sanitaria. En el actual gobierno, Sebastián Piñera, desde la presidencia, ha sido explícito en señalar su falta de acuerdo con esta y con cualquier iniciativa dirigida a ampliar las fronteras de la regulación del aborto en Chile. Junto con ello, el actual ministro de Salud, Emilio Santelices, ha dado señales inequívocas de obstaculización de la actual ley al intentar cambiar el protocolo de objeción de conciencia ${ }^{32}$, protegiendo ideologías conservadoras e intereses económicos de prestadores privados por encima de los derechos y garantías de acceso de las mujeres chilenas a los servicios. Recientemente se ha anunciado la intención de transformar el Fondo Nacional de Salud (FONASA) en el seguro público, en competencia con los seguros privados ${ }^{33}$. Esta es una señal más de una lógica que disfraza con un discurso de mayor calidad la vulneración de componentes centrales de nuestra red pública de atención de salud, (red con afiliación territorial, niveles de complejidad con coordinación real, preponderante identidad pública del sistema con lógica de protección universal y solidaria, mecanismos de participación ciudadana) ${ }^{34}$. Esta transformación, que nos acercaría al modelo colombiano y sus consecuencias ${ }^{35}$, implica una profundización de las inequidades en salud y en particular de las desigualdades de género. Nuestro sistema público de salud, pese a las dificultades estructurales comentadas al inicio, sigue siendo uno de los mejores sistemas de salud de la región. En contrapartida, la agenda de género del actual gobierno muestra la cortedad de la mirada respecto de las inequidades de género, con un listado donde se combinan declaraciones desprovistas de contenido con medidas específicas que traslucen la parcialidad en el abordaje de problemáticas complejas, como la violencia de género, junto a otras

31. MOLINA, R., MOLINA, T., CARRASCO, X., EGUIGUREN, P. Profile of abortion in Chile, with extremely restrictive law. Open J Obstet Gynecol (3):732-38. 2013.

32. El 9 de mayo de 2018 la Contraloría General de la República emitió su dictamen sobre un nuevo protocolo en relación a la ley 21.030, tras la presentación del requerimiento por un grupo de parlamentarios y organizaciones. El dictamen concluyó con que el nuevo reglamento no se ajustaba a derecho por contravenir lo dispuesto en el artículo 9 de la Constitución chilena, referido a las acciones y obligaciones del Estado para la protección de la salud.

33. Ministerio de Salud. Minuta Comisión de Acuerdo Nacional por una Salud Digna y Oportuna. Propuestas Modernización de Fonasa, Agosto 2018 https://drive.google.com/file/d/1J0bd3rZOxkPameMnHW0VFJQA11s8oSP/view [en línea].

34. VÁZQUEZ, ML., VARGAS, I., FARRE, J., TERRAZA, R. Organizaciones sanitarias integradas: Una guía para el análisis. Rev. Esp. Salud Pública. 2005; 79: 633-43.

35. Entrevista con Saúl Franco. 2012 http://viva.org.co/documentos/doc_download/145entrevista-saul-franco-ens [en línea]. 
cuyo énfasis trasluce sexismo, como la centralidad que muestra en el conjunto de medidas el matrimonio y la maternidad.

Las desigualdades de género estructurales impiden en nuestro país el goce igualitario del derecho a la salud. La debida protección de este derecho por parte del Estado ha registrado debilidad y superficialidad, aun en los periodos de Bachelet, donde se registró mayor sensibilidad y propositividad. A nivel de sistema de salud se requieren cambios estructurales hacia un sistema público universal y solidario, con fortalecimiento de la red pública y mayor integración con atención primaria. En ámbitos de protección del ejercicio de derechos sexuales y reproductivos hay acciones que los vulneran, profundizando las desigualdades de género. El rol del Estado y de la política pública como desarticuladores de la desigualdad entre géneros debe comenzar por desmontar el paternalismo y control sobre las mujeres, acabar con el utilitarismo frente a los mandatos de género sobre el cuidado, terminar con el control sobre sus cuerpos y contribuir a la construcción de autonomía en un ámbito de decisiones informadas. Debe promover la erradicación del sexismo en la interpretación de sus necesidades y terminar con la imposición de una moral del Estado-que es en realidad la de la Iglesia y el conservadurismo- sobre las libertades y propia moral de las mujeres. La laicidad efectiva del Estado es requisito sine qua non para que la protección de la salud no discrimine por género. 